\title{
КОМБИНИРОВАННАЯ АНЕСТЕЗИЯ С ИНТРАТЕКАЛЬНЫМ ВВЕДЕНИЕМ НИЗКИХ ДОЗ БУПИВАКАИНА И МОРФИН-СПИНАЛА В АБДОМИНАЛЬНОЙ ХИРУРГИИ
}

\author{
КОНОНКОВ В.В. ${ }^{1}$, МАРОЧКОВ А.В. ${ }^{2}$ \\ ${ }^{1}$ Могилевская больница №1, г. Могилев, Республика Беларусь \\ ${ }^{2}$ Могилевская областная больница, г. Могилев, Республика Беларусь \\ Вестник ВГМУ. - 2019. - Том 18, №3. - С. 45-51.

\section{COMBINED ANESTHESIA WITH INTRATHECAL ADMINISTRATION OF LOW DOSES OF BUPIVACAIN AND MORPHINE-SPINAL IN ABDOMINAL SURGERY}

\author{
KANANKOU V.V. ${ }^{1}$, MAROCHKOV A.V. ${ }^{2}$ \\ ${ }^{1}$ Mogilev Hospital No.1, Mogilev, Republic of Belarus \\ ${ }^{2}$ Mogilev Regional Hospital, Mogilev, Republic of Belarus \\ Vestnik VGMU. 2019;18(3):45-51.
}

\section{Резюме.}

Цель - сравнить эффективность и безопасность комбинированной анестезии с использованием низкодозовой спинальной анестезии с морфином с общей анестезией у пациентов, относящихся ко II, III классу по ASA и подвергающимся лапаротомным операциям на верхнем этаже брюшной полости.

Материал и методы. Было проведено проспективное нерандомизированное исследование. Было изучено и разделено на 2 группы 32 пациента в возрасте 50-88, которым выполнялись операции на органах брюшной полости по плановым показаниям. Первую группу составили пациенты, которым перед общей анестезией вводили 5 мг бупивокаина, 200 мкг морфина интратекально. Выполнено 16 низкодозовых спинальных анестезий с морфином и гипербарическим бупивокаином как компонентами комбинированной анестезии. Вторую - пациенты которым нейроаксиальные методики не применялись.

Результаты. Статистически достоверное $(\mathrm{p}<0,001)$ уменьшение количества наркотических аналгетиков в первой группе связано с сенсорным блоком в зоне операции. Достоверно снизилось количество системных опиоидов в послеоперационном периоде ( $<0,001)$, что объясняется длительным обезболивающим эффектом морфина. И в первой, и во второй группе отмечались незначительные колебания средних значений с АД (среднее АД) и ЧСС во все периоды наблюдения. Значимо меньшими данные показатели были в первой группе в момент разреза ( $<<0,01$ и 0,02$)$, что можно расценить как более эффективную ноциоцептивную защиту.

Заключение. Комбинированная анестезия с субарахноидальным введением низких доз бупивокаина и морфина позволяет эффективнее контролировать гемодинамику по сравнению с общей анестезией, снизить количество используемых системных опиоидов и миорелаксантов, уменьшить время между окончанием операции и экстубацией пациентов, с сохранением высокого уровня безопасности. Наша методика показала качественный уровень послеоперационного обезболивания со значительно меньшим использованием системных опиоидов.

Ключевые слова: гипербарический бупивокаин, интратекальный морфин, низкодозовая спинальная анестезия, абдоминальная хирургия, комбинированная анестезия.

\footnotetext{
Abstract.

Objectives. To compare the effectiveness and safety of combined anesthesia with the use of low dose hyperbaric bupivacain and morphine spinal anesthesia with general anesthesia in patients of ASA grade II and III scheduled for laparatomic operations on the organs of the upper part of the abdominal cavity.

Methods. A nonrandomized prospective trial included 32 patients aged from 50 to 88 years, who were scheduled for operation on the organs of the abdominal cavity. The first group consisted of 16 patients with subarachnoid administration of low dose hyperbaric bupivacain and morphine as the components of combined anesthesia. The second group included
} 
16 patients with general anesthesia.

Results. Statistically significant differences $(\mathrm{p}<0.001)$ of the use of the narcotic analgesics due to sensory block in the operation area were revealed. The use of systemic opioids in the postoperative period decreased credibly because of the prolonged analgetic effect of intrathecal morphine. There were minor fluctuations in the mean values of MAP and the heart rate in all periods of observation both in the first and in the second groups. These indicators were significantly lower in the first group at the time of the incision $(\mathrm{p}<0.01$ and 0.02$)$, which can be regarded as a more effective nocioceptive protection.

Conclusions. Combined anesthesia with subarachnoid administration of low doses of bupivacain and morphine allows to better control hemodynamics compared to general anesthesia, to decrease the amount of used systemic opioids and muscle relaxants, to reduce the time between the end of surgery and the extubation of patients, while maintaining a high level of safety. Our technique has shown a qualitative level of postoperative analgesia with significantly less use of systemic opioids.

Key words: hyperbaric bypivacain, intrathecal morphine, low dose spinal anesthesia, abdominal surgery, combined anesthesia.

Основная задача анестезиологии - обеспечение безопасности и комфорта пациента во время анестезии - заставляет постоянно искать решения все новых вызовов, которые ставит перед нами современная высокотехнологичная медицина. В настоящее время в анестезиологической практике во всем мире все чаще используется низкодозовая СМА (НСМА) как альтернатива общей анестезии либо стандартной СМА (спинномозговая анестезия) [1-6]. Многие авторы подтверждают безопасность данной методики у пациентов с патологией ССС (сердечно сосудистой системы) [1]. Пожилые пациенты, относящиеся ко II, III классу по ASA, являются основным контингентом общехирургических отделений. И в связи с этим разработка эффективной и безопасной методики анестезии для них является важной задачей современной анестезиологии. Гемодинамическая стабильность при эффективном уровне аналгезии является несомненным преимуществом данной методики. для пациентов с сопутствующей патологией $[1,6]$. Однако низкая управляемость НСМА, эффект присутствия, недостаточная релаксация пациента, снижающая необходимый комфорт для хирурга, ограничивают применение НСМА в абдоминальной хирургии. Мы предположили, что сочетание НСМА с общей анестезией позволит уменьшить недостатки обеих методик, сохранив их достоинства. В частности, снижение дозы фентанила и промедола, введенных интраоперационно и в послеоперационном периоде, уменьшат количество дозозависимых побочных эффектов последних [7] и позволят ускорить экстубацию и активизацию пациентов. Для проведения НСМА был выбран гипербарический бупивакаин в комбинации с морфином. Гипербаричный раствор бупивакаина позволял добиться необходимого уровня анестезии путем изменения положения тела пациента, а морфин-спинал обеспечивал послеоперационную аналгезию длительностью до 30 часов (инструкция к применению).

Цель - сравнить эффективность и безопасность комбинированной анестезии с использованием НСМА с морфином в сравнении с общей анестезией, у пациентов, относящихся ко II, III классу по ASA и подвергающихся лапаротомным операциям на верхнем этаже брюшной полости.

\section{Материал и методы}

С июня по август 2018 года на базе У3 «Могилевская больница №1» проведено проспективное нерандомизированное исследование. На проведение исследования получено разрешение комитета по зтике за №1 от 1.042018. Мы провели 32 низкодозовые спинальные анестезии с морфином и гипербарическим бупивакаином как компонентами комбинированной анестезии. Были изучены и разделены на 2 группы пациенты в возрасте 50-88 лет (табл. 1), которым выполнялись операции на органах брюшной полости по плановым показаниям (табл. 2).

Критерии включения в исследование: показания к плановому оперативному вмешательству, требующее проведения верхнесрединной лапаротомии; лица обоего пола, относящиеся ко II или III классу ASA; наличие письменного информированного согласия с методом предложенного обезболивания.

Критерии исключения из исследования: наличие абсолютных противопоказаний к прове- 
дению спинальной анестезии; отказ пациента от предложенного метода анестезии.

Пациентам выполнялись реконструктивные операции на желчных протоках, поджелудочной железе, печени, герниопластики вентральных грыж (табл. 2). Первую группу составили пациенты, которым перед общей анестезией вводили 5 мг бупивакаина, 200 мкг морфина интратекально. Вторую - пациенты которым нейроаксиальные методики не применялись. Для премедикации использовали Sol.Atropini 0,1\%-0,5ml в/в за 5 минут до анестезии. Исходный статус по ASA 2,3 класс; гемодинамику оценивали неинвазивным методом, мониторировали сАД (1/3 разницы сист. АД и диаст. АД+диаст АД), ЧСС, $\mathrm{SpO}_{2}$, ЭКГ, механику дыхания(экскурсию грудной клетки, дыхательный объем, частоту дыхания, давление на вдохе, соотношение вдоха к выдоху), концентрацию газов на вдохе и выдохе. Параметры мониторировались в течение всей анестезии. Мониторируемые параметры фиксировали: исходно (1 этап), затем после интубации (2 этап), после разреза (3 этап), через 60 мин после операции (4 этап) и во время ушивания п/операционной раны (5 этап). Лактат и гликемию контролировали в конце операции.

Для профилактики инфекции за 30 мин до разреза вводили 2000мг цефазолина. СМА проводили на уровне L2-L3 иглой Quinke 25 или 26 в положении сидя. Интратекально вводили Sol. Bupivacaine heavy 0,5\% - 1,0 мл (5 мг) и Sol. Morphine spinal 0,1\% - 0,2 ml (200 мкг). Затем пациенту придавалось положение Тренделенбурга на 5 мин., контролировались сенсорный (холодовая проба) и моторный (Bromage) блоки. Индукция в обеих группах включала: фентанил 1 мкг/кг, пропофол 2 мг/кг и 1-2 мг/кг сукцинилхолина. После введения дитилина производилась интубация трахеи и далее проводилась ИВЛ аппаратом МК1-2. Поддерживали анестезию 1\%$2 \%$ изофлюраном, фентанилом, атракуриомом. Доза гипнотика, анальгетика и миорелаксанта определялась анестезиологом путем комплексного анализа клинических параметров показателей гемодинамики, механики дыхания, рефлексов и периода полувыведения препаратов. Интраоперационную инфузию проводили кристаллоидами со V-5-6 мл/кг/час, в случае возникновения гипотензии назначался болюс кристаллоидов 10-20 мл/кг, при неэффективности титровались вазопрессоры. Гипотензия расценивалась как снижение исходного сАД на $20 \%$ и более, соот- ветственно гипертензия-повышение сАД на 20\% и более от исходного. Брадикардия определялась при снижении ЧСС ниже 50 уд. в 1 мин. Вентиляцию проводили с контролем по объему 10 мл/ кг и частотой, поддерживающей $\mathrm{CO}_{2}$ на выдохе 30-35 мм. Анестезию расценивали как недостаточную при увеличении ср АД и ЧСС более $20 \%$ в течение более 5 мин. В этом случае пациенту добавляли болюс фентанила 0,5 мкг/кг. Недостаточную релаксацию фиксировали при увеличении давления на вдохе более $20 \%$ и купировали введением атракуриума в дозе 0,15 мг/кг.

В послеоперационном периоде всем пациентам назначалась глюкозо-солевая смесь в объеме 3 мл/кг/сутки. Послеоперационное обезболивание включало в/венное введение метамизола по 1000 мг через 8 часов, кетопрофен по 100 мг через 12 часов и промедол по требованию (Боль по ВАШ >4-5 баллов). Всем пациентам назначали нмг (низкомолекулярные гепарины) в качестве тромбопрофилактики и омепразол для снижения секреции желудка. В послеоперационном периоде оценивали уровень боли по ВАШ (визуально аналоговой шкале), ПОТР (послеоперационная тошнота, рвота), головную боль, наличие депрессии дыхания, зуд, наличие аллергических реакций, осиплость голоса, гипер/гипотонию, транзиторное нарушение мочеиспускания.

Статистическую обработку полученных результатов проводили с использованием программы Statistica 7.0. Соответствие распределения нормальному определяли по критерию ShapiroWilk`s test. Данные, полученные интраоперационно, представлены медианой и нижней, и верхней квартилей. Данные ВАШ, количество промедола в послеоперационном периоде и время в ОАиР (отделение анестезиологии реанимации) для наглядности представлены в виде средних показателей значений, среднеквадратичного отклонения. Для сравнения между группами использовали критерий Манна-уитни. Для сравнения категориальных величин использовали критерий $\chi^{2}$.

\section{Результаты}

У всех пациентов были проведены успешные хирургические вмешательства. Тяжелых осложнений анестезий не было. Группы пациентов не отличались по возрасту $(p>0,1)$ Это были в основном пожилые пациенты преимущественно женского пола. Индекс массы тела в обеих группах превышал норму и соответствовал ожирению 
первой степени, различий не было ( $p>0,1)$ Семь человек в первой и восемь во второй группе относились ко второму классу, все остальные к третьему по ASA(p>0,05**) (табл. 1).

Пациенты первой группы на 20 мин ранее доставлялись в операционную для выполнения спинальной анестезии. Ни у одного из пациентов технических сложностей с CMA не отмечено. Сенсорный блок до уровня Th 3-4, моторный максимальный 1 по Бромейдж (блок в области тазобедренного сустава).

Пациенты не отличались по объему и характеру оперативных вмешательств.

Произведенные операции отражены в таблице 2.

Основными причинами, обусловившими класс тяжести, были: АГ-8 первой и 7 во второй группах, ИБС и НЖО. Значимых различий в группах не отмечено (табл. 3).

Среднее АД и ЧСС представлены в таблице 4.

Таблица 1 - Характеристика пациентов

\begin{tabular}{|c|c|c|c|}
\hline & Гр 1 & $\Gamma \mathrm{p} 2$ & $\mathrm{p}$ \\
\hline Пол, м/ж & $7 / 9$ & $5 / 11$ & $>0,05^{* *}$ \\
\hline Возраст & $64(58 ; 74)$ & $64,5(52 ; 76)$ & $>0,1^{*}$ \\
\hline ВMI & $33,5(32 ; 35)$ & $32,5(32 ; 34)$ & $>0,1^{*}$ \\
\hline ASAII/III & $7 / 9$ & $8 / 8$ & $>0,05^{* *}$ \\
\hline
\end{tabular}

Примечание: * - для анализа количественных данных использовали критерий Манна-Уитни (Mann-Whitney U-test); ** - для анализа категориальных данных использовали $\chi^{2}$ по Пирсону (Pearson Chi-square).

Таблица 2 - Характер операций

\begin{tabular}{|l|c|c|c|c|}
\hline & Гр 1 & Гр2 & $\chi^{2}$ & $\mathrm{p}$ \\
\hline Безнатяжительная герниопластика & 2 & 4 & 0,82 & $\mathrm{p}>0,05$ \\
\hline $\begin{array}{l}\text { Дистальная резекции поджелудочной железы } \\
\text { с сохранением селезенки }\end{array}$ & 1 & 0 & 1,03 & $\mathrm{p}>0,05$ \\
\hline Локальная резекция головки п.ж. по Фрей & 1 & 2 & 0,368 & $\mathrm{p}>0,05$ \\
\hline $\begin{array}{l}\text { Холедохоеюностомия на петле, отключенной } \\
\text { по РУ }\end{array}$ & 4 & 3 & 0,183 & $\mathrm{p}>0,05$ \\
\hline $\begin{array}{l}\text { Холецистэктомия. Трансдуоденпльная } \\
\text { папилосфинктеропластика }\end{array}$ & 8 & 9 & 0,125 & $\mathrm{p}>0,05$ \\
\hline
\end{tabular}

Таблица 3 - Сопутствующие заболевания

\begin{tabular}{|l|c|c|c|c|}
\hline & $\Gamma \mathrm{p} 1$ & $\Gamma \mathrm{p} 2$ & $\chi^{2}$ & $\mathrm{p}$ \\
\hline АГЗ риск 4 & 8 & 7 & 0,125 & $\mathrm{p}>0,05$ \\
\hline ИБС & 9 & 8 & 0,125 & $\mathrm{p}>0,05$ \\
\hline СД & 3 & 4 & 0,183 & $\mathrm{p}>0,05$ \\
\hline НЖО & 6 & 8 & 0,5 & $\mathrm{p}>0,05$ \\
\hline Хронический пиелонефрит & 1 & 0 & 1,03 & $\mathrm{p}>0,05$ \\
\hline Гипотиреоз & 1 & 0 & 1,03 & $\mathrm{p}>0,05$ \\
\hline Хронический бронхит & 0 & 1 & 1,03 & $\mathrm{p}>0,05$ \\
\hline
\end{tabular}


Таблица 4 - Характер гемодинамики

\begin{tabular}{|c|c|c|c|}
\hline с АД (этап операции) & Гр 1 & Гр2 & $\mathrm{p}$ \\
\hline 1 & $92(85 ; 97)$ & $96(90 ; 106)$ & $>0,1$ \\
\hline 2 & $107(95 ; 114)$ & $104(92 ; 118)$ & $>0,1$ \\
\hline 3 & $90(82 ; 99)$ & $104(92 ; 117)$ & 0,01 \\
\hline 4 & $87(80 ; 95)$ & $88(82 ; 93)$ & $>0,1$ \\
\hline 5 & $84(78 ; 88)$ & $86(82 ; 94)$ & $>0,1$ \\
\hline ЧСС (этап операции) & & & $>0,1$ \\
\hline 1 & $82(62 ; 98)$ & $87(67 ; 95)$ & $>0,1$ \\
\hline 2 & $84(77 ; 91)$ & $79(68 ; 90)$ & 0,02 \\
\hline 3 & $80(74 ; 86)$ & $92(75 ; 99)$ & $>0,1$ \\
\hline 4 & $82(76 ; 87)$ & $85(73 ; 92)$ & $>0,1$ \\
\hline 5 & $79(70 ; 92)$ & $78(72 ; 81)$ & $>0,1$ \\
\hline лактат & $0,67(0,35 ; 1,22)$ & $0,74(0,52 ; 1,15)$ & $>0,1$ \\
\hline
\end{tabular}

Таблица 5 - Этап анестезии

\begin{tabular}{|c|c|c|c|}
\hline & Гр 1 & $\Gamma \mathrm{p} 2$ & $\mathrm{p}$ \\
\hline Время операции, мин & $109(72 ; 137)$ & $105(69 ; 135)$ & $>0,1$ \\
\hline Инфузия, мл & $1050(707 ; 1340)$ & $1020(610 ; 1270)$ & $>0,1$ \\
\hline Вазотоники & 1 & & 0,001 \\
\hline Фентанил, мг & $0,2(0,15 ; 0,35)$ & $0,35(0,31 ; 0,41)$ & 0,003 \\
\hline Атракуриум, мг & $36,1(24,2 ; 60,2)$ & $48,5(39,1 ; 62,2)$ & 0,001 \\
\hline$\wedge$ t, мин & $12(11 ; 20)$ & $21(15 ; 55)$ & \\
\hline
\end{tabular}

Таблица 6 - Послеоперационный этап

\begin{tabular}{|c|c|c|c|}
\hline & Гр 1 & Гр2 & $\mathrm{p}$ \\
\hline ВАШ (через 60 мин) & $3,2+-1,14$ & $3,3+-1,34$ & $>0,1$ \\
\hline ВАШ (через 180 мин) & $4,3+-1,55$ & $4,9+-2,21$ & $>0,1$ \\
\hline ВАШ (через 24 часа) & $4,4+-2,74$ & $4,9+-2,17$ & $>0,1$ \\
\hline Промедол, мГ & $7,2+-7,12$ & $37,5+-7,27$ & 0,001 \\
\hline Часы в ОАиР & $22,9+-22,8$ & $23,2+-20,1$ & $>0,1$ \\
\hline Выписан через дней & $11,9+-6,2$ & $11,3+-7,2$ & $>0,1$ \\
\hline
\end{tabular}

Таблица 7 - Осложнения

\begin{tabular}{|l|c|c|c|c|}
\hline & Г 1 & $\Gamma \mathrm{p} 2$ & $\chi^{2}$ & $\mathrm{p}$ \\
\hline $\begin{array}{l}\text { Послеоперационная } \\
\text { тошнота, рвота }\end{array}$ & 3 & 2 & 0,627 & $\mathrm{p}>0,05$ \\
\hline Зуд & 2 & 1 & 0,545 & $\mathrm{p}>0,05$ \\
\hline Головная боль & 3 & 0 & 0,069 & $\mathrm{p}>0,05$ \\
\hline $\begin{array}{l}\text { Транзиторное нарушение } \\
\text { мочеиспускания }\end{array}$ & 2 & 0 & 0,145 & $\mathrm{p}>0,05$ \\
\hline Гипер/гипотензия & 4 & 6 & 0,446 & $\mathrm{p}>0,05$ \\
\hline Продленная ИВЛ & 0 & 2 & 0,145 & $\mathrm{p}>0,05$ \\
\hline Осиплость голоса & 0 & 1 & 0,31 & $\mathrm{p}>0,05$ \\
\hline
\end{tabular}

В послеоперационном периоде мониторировался уровень боли по ВАШ и производились отметки через 1 час, 3 и через 24 часа. При уровне боли $>5$ вводился промедол. Фиксировалось время, проведенное в ОАиР, в часах и время пребывания в стационаре - в днях (табл. 6).
Послеоперационные проблемы фиксировались у пациентов обеих групп. Наиболее частой проблемой было повышение давления, которое чаще наблюдалось у пациентов второй группы и лечилось назначением гипотензивных препаратов. Частота ПОТР в обеих группах была иден- 
тична, однако методы купирования отличались. Если во второй группе терапия ондансетроном позволила достичь $100 \%$ эффекта, то в первой одному пациенту пришлось вводить налоксон для купирования повторяющейся рвоты. Транзиторное нарушение мочеиспускания у пациентов мужского пола в первой группе были излечены временной постановкой мочевого катетера. А низкий мышечный тонус у двух пациентов второй группы привел к необходимости продленной ИВЛ в послеоперационном периоде (табл. 7).

\section{Обсуждение}

Невысокий процент вазотоников говорит о незначительном влиянии низкодозовой СМА на гемодинамику пациентов со значительным коморбидным фоном в условиях комбинированной анестезии. Статистически достоверное $(\mathrm{p}<0,001)$ уменьшение количества наркотических аналгетиков в первой группе связано с сенсорным блоком в зоне операции. Механизм снижения количества мышечных релаксантов $(\mathrm{p}<0,003)$ не совсем ясен и требует дальнейшего изучения. Значительно $(p>0,1)$ меньше было ^t (мин) в первой группе, по-видимому, это обусловлено меньшим количеством использованных опиоидов и релаксантов. Достоверно снизилось количество системных опиоидов в послеоперационном периоде $(\mathrm{p}<0,001)$, что объясняется длительным обезболивающим эффектом морфина. И в первой, и во второй группе отмечались незначительные колебания средних значений сАД и ЧСС во все периоды наблюдения. Значимо меньшими данные показатели были в первой группе в момент разреза $(\mathrm{p}<0,01$ и 0,02$)$, что можно расценить как более эффективную ноциоцептивную защиту. Нормальный уровень гликемии и лактата в раннем послеоперационном периоде говорит об удовлетворительной тканевой перфузии, что подтверждает хорошее качество анестезиологического обеспечения пациентов обеих групп. Общий уровень послеоперационного обезболивания в обеих группах не отличался, однако в первой группе понадобилось значительно меньше опиоида $(\mathrm{p}<0,001)$. Частота осложнений ни в одной из групп статистически не отличалась и соответствовала таковым у других исследователей $[8,9,10]$. Время, проведенное пациентами в ОАиР и стационаре, существенно не отличалось.

\section{Заключение}

1. Комбинированная анестезия с субарахноидальным введением низких доз бупивакаина и морфина позволяет эффективнее контролировать гемодинамику по сравнению с общей анестезией.

2. Снизить количество используемых системных опиодов и миорелаксантов, уменьшить время между окончанием операции и экстубацией пациентов с сохранением высокого уровня безопасности.

3. Наша методика показала качественный уровень послеоперационного обезболивания со значительно меньшим использованием системных опиоидов.

\section{Литература}

1. Watson, B. Spinal anaesthesia a practical guide / B. Watson, J. Allen, I. Smith. - Norwich : Colman Print, 2004. - 17 p.

2. Comparison of low dose spinal anesthesia with general anesthesia in pre-eclamptic parturients undergoing emergency cesarean section / A. G. Sivevski [et al.] // APICare. - 2015 Jan. - Vol. 19, N 1. - P. 37-43.

3. Sinha, R. Laparoscopic cholecystectomy under spinal anesthesia: a study of 3,492 patients / R. Sinha, A. K. Gurwara, S. C. Gupta // J. Laparoendosc. Adv. Surg. Tech. A. -2009 Jun. - Vol. 19, N 3. - P. 323-327.

4. Koju, R. B. Effectiveness of Spinal Anaesthesia versus General Anaesthesia for Open Cholecystectomy / R. B. Koju, Y. Dongol, R. Verma // J. Nepal. Health. Res. Counc. - 2016 May. - Vol. 14, N 33. - P. 93-98.

5. Khan, M. N. Spinal anesthesia versus general anesthesia for open cholecystectomy: comparison of postoperative course / M. N. Khan, M. N. Ashraf, H. D. Khan // Ann. Pak. Inst. Med. Sci. - 2013. - Vol. 9, N 2. - P. 95-98.

6. Laparoscopic cholecystectomy under segmental thoracic spinal anesthesia: a feasibility study / A. A. van Zundert [et al.] // Br. J. Anaesth. - 2007 May. - Vol. 98, N 5. - P. 682-686.

7. Рациональная фармакоанестезиология : рук. для практикующих врачей / под общ. ред. А. А. Бунятяна, В. М. Мизикова. - М. : Литтера, 2006. - 800 с.

8. Low dose spinal anesthesia for open cholecystectomy: a feasibility and safety study / J. Laoutid [et al.] // Int. Surg. J. - 2017. - Vol. 4, N 4. - P. 1417-1421.

9. Thune, A. Prevention of postoperative nausea and vomiting after laparoscopic cholecystectomy. A prospective randomized study of metoclopramide and transdermal hyoscine / A. Thune, L. Appelgren, E. Haglind // Eur. J. Sur. - 1995 Apr. - Vol. 161, N 4. - P. 265-268.

10. Al-Shraideh, A. Low Dose Spinal Anaesthesia in Elderly \& Critically Ill Patients / A. Al-Shraideh // J. Anesth. Crit. Care. - 2015. - Vol. 3, N 3. - P. 00099. 


\section{References}

1. Watson B, Allen J, Smith I. Spinal anaesthesia a practical guid. Norwich: Colman Print; 2004. 17 p.

2. Sivevski AG, Sholjakova MV, Kartalov AB, Kuzmanovska BK, Durnev VM. Comparison of low dose spinal anesthesia with general anesthesia in pre-eclamptic parturients undergoing emergency cesarean sectionю. APICare. 2015 Jan;19(1):37-43.

3. Sinha R, Gurwara AK, Gupta SC. Laparoscopic cholecystectomy under spinal anesthesia: a study of 3,492 patients. J Laparoendosc Adv Surg Tech A. 2009 Jun;19(3):323-7. doi: 10.1089/lap.2008.0393

4. Koju RB, Dongol Y, Verma R. Effectiveness of Spinal Anaesthesia versus General Anaesthesia for Open Cholecystectom. J Nepal Health Res Counc. 2016 May;14(33):93-98.

5. Khan MN, Ashraf MN, Khan HD. Spinal anesthesia versus general anesthesia for open cholecystectomy: comparison of postoperative course. Ann Pak Inst Med Sci. 2013;9(2):95-8.
6. van Zundert AA, Stultiens G, Jakimowicz JJ, Peek D, van der Ham WG, Korsten HH, et al. Laparoscopic cholecystectomy under segmental thoracic spinal anesthesia: a feasibility study. Br J Anaesth. 2007 May;98(5):682-6. doi: 10.1093/ bja/aem058

7. Bunyatyan AA, Mizikov VM, red. Rational pharmacoanesthesiology: ruk dlia praktikuiushchikh vrachei. Moskva, RF: Littera; 2006. 800 p. (In Russ.)

8. Laoutid J, Jbili N, Kechna H, Hachimi MA. Low dose spinal anesthesia for open cholecystectomy: a feasibility and safety study. Int Surg J. 2017;4(4):1417-21. doi: 10.18203/23492902.isj20171153

9. Thune A, Appelgren L, Haglind E. Prevention of postoperative nausea and vomiting after laparoscopic cholecystectomy. A prospective randomized study of metoclopramide and transdermal hyoscine. Eur J Surg. 1995 Apr;161(4):265-8.

10. Al-Shraideh A. Low Dose Spinal Anaesthesia in Elderly \& Critically Ill Patients. J Anesth Crit Care. 2015;3(3):00099. doi: 10.15406/jaccoa.2015.03.00099

\section{Сведения об авторах:}

Кононков В.В. - заведующий отделением анестезиологии и реанимации, Могилевская больница №1, ORCID: https://orcid.org/0000-0002-0000-658X:

Марочков А.В. - д.м.н., профессор, врач-анестезиолог-реаниматолог, Могилевская областная больница, ORCID: http://orcid.org/0000-0001-5092-8315.

\section{Information about authors:}

Kanankou V.V. - head of the anesthesiology \& resuscitation department, Mogilev Hospital No.1, ORCID: https://orcid.org/0000-0002-0000-658X:

Marochkov A.V. - Doctor of Medical Sciences, professor, anesthesiologist-resuscitator, Mogilev Regional Hospital, ORCID: http://orcid.org/0000-0001-5092-8315.

Адрес для корреспонденции: Республика Беларусь, 212018, г. Могилев, ул. Ак. Павлова, д. 2, Могилевская больница №1, отделение анестезиологии и реанимации. E-mail: Valeryrao@mail.ru - Кононков Валерий Васильевич.

Correspondence address: Republic of Belarus, 212018, Mogilev, 2 Academician Pavlov str., Mogilev Hospital No.1, the anesthesiology \& resuscitation department.E-mail:Valeryrao@mail.ru-Valery V.Kanankou. 